\title{
RELACIÓN ENTRE DIFERENTES PRUEBAS DE CAMPO: FUERZA, POTENCIA Y VELOCIDAD
}

\author{
Jessenia Hernández Elizondo \\ Escuela de Educación Física y Deportes \\ Universidad de Costa Rica, San José, Costa Rica \\ E-mail: jhelizon@cariari.ucr.ac.cr
}

\begin{abstract}
Resumen
Hernández-Elizondo, J. (2003). Relación entre diferentes pruebas de campo: fuerza, potencia y velocidad. Revista de Ciencias del Ejercicio y la Salud, 3(1), 1-10. Esta investigación tuvo como propósito principal el encontrar por medio de pruebas en las variables de fuerza, potencia y velocidad, la relación matemática existente entre estos tres constructos. Participaron 56 sujetos varones con edades entre 18 y 25 años. Con el fin de responder al objetivo del estudio, se les aplicaron pruebas de campo de fuerza (1RM de sentadilla completa y repeticiones máximas al $80 \%$ 60\% 40\% y 20\%), de velocidad (30 metros lanzados y 30 metros sin impulso), y de potencia (salto vertical con contra-movimiento SVCM, salto vertical sin impulso SVSI y salto vertical con caída SVCC), para correlacionar las diferentes pruebas entre sí. Los datos fueron analizados por medio de la correlación de Pearson (r), para correlacionar parejas de pruebas y correlación canónica (Rc) para correlacionar grupos de variables. A partir de cada correlación se obtuvo la varianza explicada $\left(r^{2}\right)$ la cual indica el porcentaje de relación compartida en escala de 0-100 que existe entre las pruebas. Según los datos obtenidos, no existe relación significativa (2.1\%) entre las pruebas de salto-sentadilla, ni relación significativa $(5.1 \%)$ entre las pruebas de velocidad- sentadilla, mientras que la relación salto- velocidad $(62 \%)$, fue estadísticamente significativa $(p<0.05)$. La implicación de estos resultados podrían respaldar la idea de que la relación teórica que existe entre estos constructos, no es completamente cierta cuando éstos son medidos por medio de pruebas de campo. PALABRAS CLAVES: entrenamiento deportivo, fuerza, potencia, velocidad.
\end{abstract}

\section{INTRODUCCIÓN}

Ha sido de enorme interés y preocupación para los entrenadores, inmersos en el campo deportivo, el encontrar nuevas formas para lograr mejoramientos en la fuerza $(\mathrm{F})$, la potencia $(\mathrm{P})$ y la velocidad $(\mathrm{V})$, conceptos íntimamente ligados y relacionados. Tradicionalmente cada una de estas variables son elementos fundamentales en la preparación de cualquier deportista, como componente de apoyo para el rendimiento deportivo.

Todo entrenador conoce la fórmula $\mathrm{P}=\mathrm{F}$ $\mathrm{x} \mathrm{V}$, y desearía que su atleta, logre el mayor desempeño en estas tres variables. Sin embargo, el cómo lograrlo parece ser el punto de divorcio entre la teoría y la práctica real, ya que el conocimiento científico, ofrece herramientas que parecen estar subutilizadas, dejando gran margen de los resultados al azar y al empirismo.

Esta práctica generalizada en el medio crea un vacío de información y de procedimientos científicos, que podrían estar en función de optimizar el proceso de entrenamiento de los atletas. Es importante conocer entonces, cuáles son los mecanismos que el cuerpo humano utiliza como medios para enfrentar cada una de las situaciones motoras que experimenta cuando realiza un movimiento.

La fuerza nunca aparece en los distintos deportes bajo una forma estrictamente pura, sino por medio de una combinación de factores físicos que condicionan el rendimiento, así se puede llegar a distinguir dos manifestaciones, a saber: a) 
manifestación activa de la fuerza, la cual se refiere a la tensión que es capaz de generar un músculo por acción de una contracción muscular voluntaria, y b) manifestación reactiva de la fuerza, que es definida por Verjochanski (1990) como la capacidad de fuerza que realiza un músculo como reacción a una fuerza externa que modifica o altera su propia estructura, normalmente caracterizada por producirse tras un ciclo de estiramientoacortamiento (CEA).

En la actualidad el constructo fuerza es determinado mediante dos métodos principales, uno de ellos se da en condiciones de laboratorio con el uso de la plataforma de fuerza u otros equipos de alta tecnología, y la otra forma es por medio de pruebas de campo. Aunque la medición directa de la fuerza no es posible de realizar, ya que para que se dé, se debe aislar el músculo o a una determinada fibra muscular, lo cual es fisiológicamente imposible en humanos, mientras estén vivos, la aproximación de la medición de la fuerza se puede realizar gracias a diferentes mecanismos. El protocolo de campo más utilizado es el de IRM (una repetición máxima) que expresa la capacidad de un individuo de levantar la mayor carga posible en una única repetición (Thomas y Nelson, 1990).

Como se citó anteriormente otra cualidad motriz importante en la generación del movimiento es la velocidad, la cual es definida por García, Navarro, Ruiz y Acero (1998) como la capacidad de un sujeto para realizar acciones motoras en un mínimo de tiempo y con el máximo de eficacia. Ahora bien, dentro de lo que es velocidad, existen diferentes tipos, especificadas cada una de acuerdo a las necesidades planteadas en el momento preciso de su utilización; así, se distingue la velocidad de reacción, que es la facultad que tiene el sistema nervioso para recibir un estímulo y convertirlo en una orden motriz (Pila, 1985), este estímulo es generalmente de tipo visual, auditivo o táctil.

Otro tipo de velocidad es la de contracción, la cuál está relacionada con el tiempo que necesita cada sujeto para alcanzar diferentes niveles de fuerza. Grosser, Starischka, Zimmermann (1988) la definen como la capacidad de mantener el máximo de contracciones a la velocidad máxima posible en un determinado tiempo.

La velocidad de aceleración, se refiere a la capacidad de establecer una velocidad máxima en el menor tiempo posible, ésta debe ser continua, progresiva y definida. Y por último la velocidad máxima, que es la velocidad que comprende la etapa posterior de las antes en mención, y se refiere a la velocidad máxima alcanzada durante el gesto deportivo (Grosser y otros, 1988).

Estos diferentes tipos de velocidad son entrenables y mejorables, y existen diferentes características en el entrenamiento, como: trabajar a niveles máximos y submáximos, utilizar distancias cortas e intervalos, con manipulación de tiempos de descanso; que permiten incrementar las diferentes manifestaciones de la velocidad y así lograr una mayor velocidad total.

En el cuerpo humano, por definición, la mejora en las variables de fuerza y velocidad son indispensables para optimizar el rendimiento deportivo. Sin embargo estas cualidades físicas no se dan en forma aislada, más su combinación resulta en un constructo conocido como potencia, que es definida por Komi (1992) como la capacidad que tiene el sistema neuromuscular, para superar resistencias con la mayor velocidad de contracción posible.

De forma teórica, el músculo desarrolla la máxima potencia cuando la velocidad de acortamiento es de $1 / 3$ de la velocidad máxima de acortamiento y ésta se aplica contra una resistencia de $1 / 3$ de la fuerza máxima, pero esta afirmación puramente matemática, no coincide con los valores de fuerza y velocidad, en los cuales el músculo obtiene el máximo rendimiento mecánico. En este caso los valores correspondientes son de la $1 / 2$ de la máxima fuerza isométrica y de $1 / 5$ de la velocidad máxima. (Cavagna y Kaneco, 1976). No obstante, la determinación de esta fórmula resulta compleja de calcular en el músculo in vivo, ya que no se pueden 
amortiguar los efectos de numerosos parámetros que afectan la complejidad del movimiento humano. Así, no es conveniente asumir que multiplicando la fuerza ejercida durante una contracción por la velocidad con que se acorta, se obtiene la potencia en ese instante.

Es por esta razón que para la valoración de las diferentes cualidades físicas han sido diseñadas pruebas de campo que se relacionan en forma significativa, matemáticamente hablando, con cada uno de los constructos, (fuerza, potencia y velocidad) medidos bajo condiciones de laboratorio y en músculo aislado. A este tipo de validez se le llama validez concurrente. Un claro ejemplo es el salto vertical, el cual relaciona la altura alcanzada luego de saltar verticalmente, con el nivel de potencia de piernas del ejecutante. Mientras que en el caso de las pruebas de sentadilla y de velocidad, las pruebas son determinadas por validez lógica, que es clasificada por Thomas y Nelson (1990) cuando la prueba o medición obviamente involucra la ejecución que está siendo medida.

El entrenamiento de la potencia, se basa en el trabajo de tipo reactivo, uno de los métodos más conocidos para el mejoramiento del trabajo de tipo reactivo, es el entrenamiento pliométrico. Para Díaz y Molina (1994) el entrenamiento pliométrico es un método que posee la capacidad de mejorar, además de la fuerza, la potencia y la capacidad atlética. Esa es la razón por la que para muchos autores los ejercicios pliométricos son el método más utilizado para el desarrollo del poder explosivo de los músculos extensores de las piernas. Esta es quizás la forma de entrenamiento más intensa que se pueda practicar, teniendo la ventaja de que es fácil de enseñar y aprender, por lo que es actualmente utilizado por una gran cantidad de entrenadores y atletas.

Los ejercicios pliométricos son todos aquellos realizados con el propio peso corporal que impliquen el CEA, permitiendo también la actuación de los órganos tendinosos de Golgi y husos musculares
(Ortíz, 1996). A su vez este tipo de ejercicio mejora la capacidad para activar un mayor número de fibras musculares en el menor tiempo posible, y resultando en una mayor potencia en el atleta (Peralta, 1993).

Bosco (1985), señala que durante la ejecución, dependiendo de la intensidad y tipo de salto, se estimulan muchos cambios a nivel neuromuscular, lo cual produce cambios rápidos y poderosos a lo largo de los músculos. Según Weineck (1988), esta técnica de entrenamiento utiliza tres factores dentro del músculo: el reflejo de extensión, la pre-inervación y los componentes elásticos. En el momento del salto, se produce un aumento de fibras musculares activadas por la acción del reflejo de extensión, durante el contacto con el suelo la pre-inervación del músculo asume una participación importante por cuanto crea un fondo de inervación óptima para la actividad subsiguiente y modifica el estado de tensión (elasticidad muscular) que es responsable del tamaño y rapidez de la extensión muscular. Los componentes elásticos del músculo son utilizados como almacenadores de energía cinética, en forma de energía potencial, que posteriormente se añade a la contracción muscular concéntrica.

No obstante esa energía sólo puede ser reutilizada si el período de transición entre las fases excéntrica y concéntrica es corto, ya que conforme transcurre el tiempo, la tensión cae y la energía elástica almacenada se pierde (Miller y Power, 1982). Sin embargo Aragón (1994) afirma que la obtención de una mayor eficiencia, no necesariamente se debe al almacenamiento de energía elástica, sino que la elasticidad del tendón, podría afectar la potencia mecánica, al permitir que el acercamiento entre el origen y la inserción del músculo tenga una velocidad mayor que la velocidad de acortamiento de las fibras musculares y permitir un rango de movimiento mayor a las articulaciones, teniendo ventaja de esta manera el tendón menos rígido o más elástico.

Diversos autores (Bosco, 1994; Miller y Power, 1982; Vega, 1993) hacen referencia a 
la explicación de funcionamiento del entrenamiento pliométrico, el cual consiste en que el atleta debe subirse a una plataforma de una altura determinada y dejarse caer para que, al tocar la superficie del suelo, salte inmediatamente, aquí el peso del cuerpo y la gravedad se combinan para crear una resistencia que estresa las piernas.

Según la fórmula antes mencionada de $\mathrm{P}=\mathrm{F} \times \mathrm{V}$, matemáticamente, y desde el punto de vista puramente físico, la relación entre estos componentes es clara, si se asume que al mejorar uno de los componentes, al menos uno de los otros dos debe mejorar; sin embargo eso no necesariamente se ha dado claramente, cuando se ha traducido al ámbito deportivo

Tomando en cuenta este vacío entre la teoría y la práctica, y en busca de respuestas que permitan mejorar a nivel deportivo, se propone este estudio cuyo propósito fue: Conocer por medio de un análisis correlacional, la relación matemática entre cada una de las pruebas de campo de fuerza, potencia y velocidad.

\section{METODOLOGÍA}

\section{Participantes}

17 sujetos varones no entrenados, con edades comprendidas entre 18 y 25 años, participaron voluntariamente en esta investigación. Antes de iniciar cada participante recibió una explicación del propósito del estudio y firmó un consentimiento informado

\section{Instrumentos de medición}

$\checkmark$ Cronómetro Casio Hs-3.

$\checkmark$ Saltímetro.

$\checkmark$ Racket o soporte para barra de peso libre.

$\checkmark$ Barra de 10 libras.

$\checkmark$ Discos de: $2.5,5,10,25,35$ y 50 libras. (peso libre)

$\checkmark \quad$ Banca de $35 \mathrm{~cm}$ de alto.

\section{Pruebas utilizadas}

Tomando en consideración que la medición de la potencia y la velocidad se hacen por medio de pruebas de campo que involucran únicamente los grupos musculares de las extremidades inferiores, la valoración de la fuerza se realizó en un único ejercicio: la sentadilla completa.

Cabe señalar que todas las pruebas que se analizaron en este estudio fueron pruebas de campo. A continuación se detallan cada una de ellas:

\section{Pruebas de Fuerza:}

a. 1RM de Sentadilla completa.

b. Repeticiones máximas al $80 \%$ de 1RM.

c. Repeticiones máximas al $60 \%$ de $1 \mathrm{RM}$.

d. Repeticiones máximas al $40 \%$ de 1RM

e. Repeticiones máximas al $20 \%$ de 1 RM.

\section{Pruebas de Potencia:}

a. Salto vertical con contra movimiento (SVCM).

b. Salto vertical sin impulso (SVSI) .

c. Salto vertical con caída (SVCC).

\section{Pruebas de Velocidad:}

a. 30 metros sin impulso.

b. 30 metros con impulso o lanzados (con 20 metros lanzados).

\section{Procedimiento}

Cada sujeto se midió en forma individual en cada una de las pruebas, con tres intentos en las pruebas de velocidad y potencia.

Las pruebas fueron aplicadas en cuatro días diferentes con una semana entre cada prueba. Los sujetos descansaron durante 30 minutos entre prueba y prueba. El primer día, los sujetos realizaron la prueba de 1RM de sentadilla completa y las repeticiones máximas al $80 \%$ de 1RM. El segundo día se 
llevaron a cabo las pruebas de fuerza en los porcentajes de $60 \%, 40 \%$ y $20 \%$ de $1 \mathrm{RM}$; el tercer día de medición los sujetos realizaron las pruebas de velocidad y el cuarto día, las pruebas de potencia. En cada sesión se realizó un calentamiento general y uno específico, según las pruebas que correspondían.

\section{Análisis Estadístico}

Los procedimientos del análisis estadístico se describen a continuación:

a. Se calcularon los promedios de los intentos para cada sujeto en cada prueba de potencia y velocidad.

b. Los datos fueron analizados por medio de la correlación de Pearson (r), para correlacionar parejas de pruebas, y correlación canónica $(\mathrm{Rc})$ para correlacionar grupos de pruebas, lo que indica la relación que tienen las pruebas entre sí.

c. A partir de cada correlación, se obtuvo la varianza explicada $\left(\mathrm{r}^{2}\right)$, que indicó el porcentaje de relación compartida en escala de 0-100, que existe entre las pruebas.

\section{RESULTADOS}

A continuación se presentan los cuadros de correlación entre las variables de potencia y velocidad, con los resultados en las pruebas de fuerza (Tabla 1 y Tabla 2).

Tabla 1. Correlación de Pearson entre las variables de fuerza y la potencia.

\begin{tabular}{lccccc}
\hline & $\mathbf{1 R M}$ & $\mathbf{8 0 \%}$ & $\mathbf{6 0 \%}$ & $\mathbf{4 0 \%}$ & $\mathbf{2 0 \%}$ \\
\cline { 2 - 6 } S.S.I & 0.180 & -0.127 & 0.034 & -0.035 & 0.206 \\
S.C.I & 0.073 & -0.138 & -0.187 & -0.243 & 0.060 \\
S.C.C & 0.106 & -0.050 & -0.008 & -0.039 & 0.312 \\
\hline
\end{tabular}

$* \mathrm{r}$ tabular $=0.514 ; \alpha=0.05$

(Valor mínimo para que una relación sea estadísticamente significativa).

Tabla 2. Correlación de Pearson entre las variables de velocidad y fuerza.

\begin{tabular}{lccccc}
\hline & 1RM & $\mathbf{8 0 \%}$ & $\mathbf{6 0 \%}$ & $\mathbf{4 0 \%}$ & $\mathbf{2 0 \%}$ \\
\cline { 2 - 6 } 30 m lanz. & -0.292 & -0.126 & 0.011 & -0.158 & -0.450 \\
30 m sin & -0.164 & 0.225 & 0.100 & 0.242 & -0.200 \\
\hline
\end{tabular}

$* \mathrm{r}$ tabular $=0.514 ; \alpha=0.05$

(Valor mínimo para que una relación sea estadísticamente significativa).

Estas tablas nos indican que en ninguna de las relaciones se presentó una correlación con valores altos, por lo que se deduce que no existe una correlación significativa entre las pruebas de fuerza y las pruebas de potencia y velocidad.

La tabla 3 ilustra la relación entre las pruebas de potencia y de velocidad.

Tabla 3. Correlación de Pearson entre las variables de potencia y velocidad.

\begin{tabular}{lccc}
\hline & \multicolumn{3}{c}{ Tipo de salto } \\
\cline { 2 - 4 } & Con impulso & Sin impulso & Con caída \\
\cline { 2 - 4 } 30 m lanzados & $-0.818^{* *}$ & $-0.538^{*}$ & $-0.821^{* *}$ \\
$\mathbf{3 0}$ m sin impulso & $-0.852^{* *}$ & $-0.866^{* *}$ & $-0.810^{* *}$ \\
\hline
\end{tabular}

$$
* \mathrm{p}<0.05 ; * \mathrm{p}<0.01
$$


Este cuadro de matriz de correlación entre las pruebas de velocidad y potencia muestra que todas las relaciones son significativas y de signo negativo, lo cual indica que a mayor potencia (medida en unidades centímetros), mayor velocidad (medida en unidades de segundos).
Además se obtuvo el análisis de varianza explicada $\left(\mathrm{r}^{2}\right)$ promedio entre los tres grupos de constructos (ver diagrama $\mathrm{n}^{\mathrm{o}} 1$ ) para explicar el porcentaje de varianza compartida en escala de $0-100$.

\section{Diagrama 1. Varianza compartida entre las variables de fuerza, potencia y velocidad.}

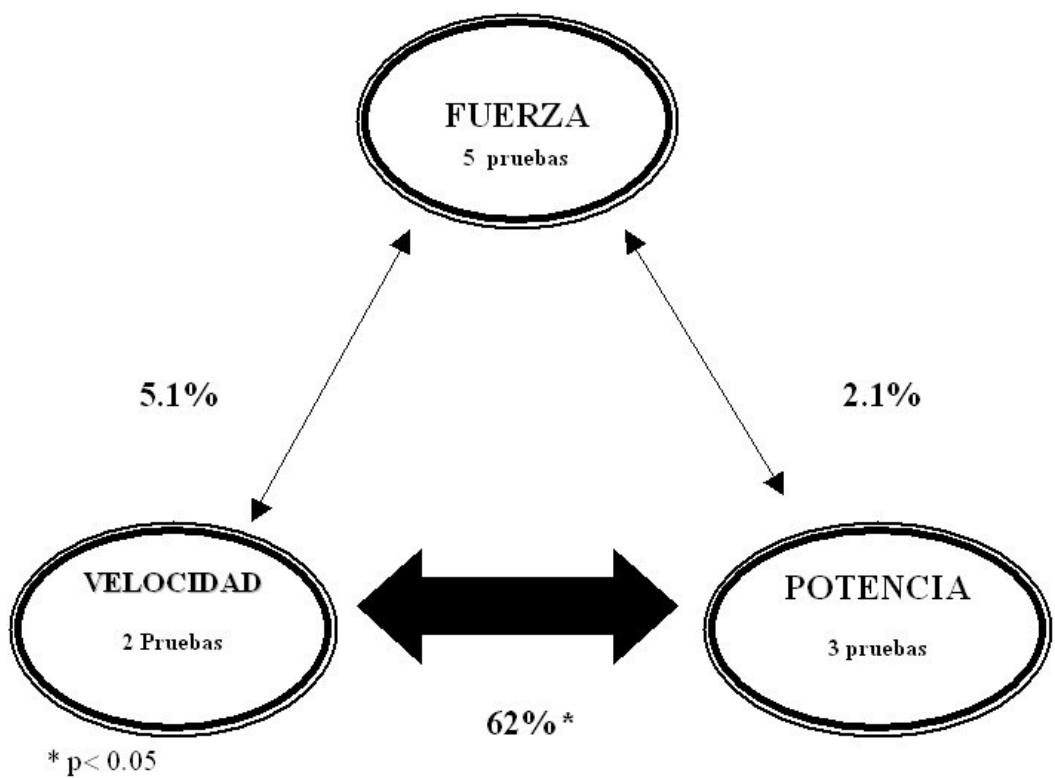

Se demuestra claramente que entre los constructos de fuerza y de potencia, existe un porcentaje de relación de tan sólo un $2,1 \%(\mathrm{Rc}=0.14)$, para los constructos de fuerza y velocidad la relación sigue siendo no significativa $(\mathrm{Rc}=0.22)$, ya que el porcentaje de varianza explicada es de $5,1 \%$. Sin embargo la relación potencia-velocidad es altamente significativa siendo ésta de un $62 \%$ $(\mathrm{Rc}=0.79)$.

\section{DISCUSIÓN}

Si bien es cierto, el ámbito deportivo se abre cada día más hacia el conocimiento probado científicamente, muchas de las personas que están inmersas en él, siguen absorbiendo información teórica y llenando sus inquietudes, por medio de conocimientos previamente establecidos, sin hacer ningún tipo de cuestionamiento, o buscar alternativas que puedan optimizar el rendimiento de sus deportistas.

Este comportamiento, de aceptación pasiva, ha provocado que inevitablemente, sean los atletas, quienes pagan los errores y la pérdida de tiempo en su preparación, causada por el impedimento cultural y académico, tanto de ellos mismos como de sus entrenadores, lo que les limita las posibilidades de cuestionar los lineamientos teóricos que hoy por hoy dirigen el entrenamiento deportivo.

Tomando en cuenta este vacío entre la literatura y la práctica, se propuso el presente estudio, el cual como propósito 
principal fue conocer por medio de un análisis correlacional, la relación matemática entre los constructos de fuerza, potencia y velocidad, medidos éstos por medio de pruebas de campo.

Los resultados obtenidos en el estudio se encuentran resumidos en las tablas $1,2,3$ y en el diagrama 1 , previamente descritos.

$\mathrm{Si}$ se observa, el diagrama 1 , se puede notar, que la tendencia en los resultados de la relación sentadilla - salto, es no significativa $(2.1 \%)$ Por otro lado el mismo diagrama muestra un comportamiento muy débil $(5.1 \%)$ y estadísticamente no significativo en la relación sentadilla carreras de velocidad, sin obviar o despreciar que existe una relación porcentual entre ambos grupos de pruebas. Contrario a lo que presentan las combinaciones anteriores, la relación matemática entre las pruebas de salto - carreras de velocidad, es mayormente consistente y estadísticamente significativa
(62\%), lo cuál concuerda con Baker y Nance (1999) quien encontró una relación mayor entre las pruebas de velocidad y potencia que entre las de velocidad y fuerza, luego de realizar un estudio en el que utilizó pruebas de campo para el tren inferior en jugadores profesionales de rugby.

Puesto que la relación porcentual, se presenta en todos los grupos de pruebas, es difícil buscar explicaciones concluyentes acerca de estos datos, los cuales, son de gran importancia para la optimización en el campo del entrenamiento deportivo.

Las características de movimiento en cada una de las pruebas realizadas, pueden ser el pilar común que, las diferentes combinaciones que se presentan en el párrafo anterior, aprovechan para que una se relacione o no con la otra. A continuación, se presenta una tabla de características mecánicas y fisiológicas, en los diferentes tipos de pruebas realizadas (Tabla 4):

Tabla 4. Resumen de características mecánicas y fisiológicas de diversas pruebas.

\begin{tabular}{lccc}
\hline \multirow{2}{*}{ Característica } & \multicolumn{3}{c}{ Pruebas o Tests } \\
\cline { 2 - 4 } & Sentadilla Completa & Salto & Velocidad \\
\hline Carga & (Externa) doble del peso corporal & Propio peso corporal & Propio peso corporal \\
Desplazamiento & Vertical & Vertical & Horizontal \\
Velocidad de movimiento & Lenta & Rápida & Rápida \\
Apoyo en el piso & Ambas piernas & Ambas piernas & Pierna alternada \\
Angulo de flexión & mayor & medio & medio \\
Tipo de contracción & Ciclo estiramiento-acortamiento & Ciclo estiramiento- & Ciclo estiramiento- \\
& & acortamiento & acortamiento \\
\hline
\end{tabular}

En el cuerpo humano, las acciones motoras generalmente están dadas por movimientos complejos o multiarticulares y en este caso, las diferentes pruebas realizadas no son la excepción, es de suma importancia tomar en cuenta que este tipo de movimiento, provoca acciones diferentes y contrarias en los músculos, huesos y tendones involucrados. Por ejemplo la fase positiva de un salto vertical (cuando las articulaciones se están extendiendo) el gastronemio tiende a acortarse al producir flexión plantar, pero tiende a alargarse en virtud de la extensión de rodilla. (Bobbert, Huijing, y Gerrit Jan Van, 1986) entendiendo, entonces que el análisis de la acción muscular es sumamente complejo, y difícil de explicar.

Las características mecánicas y fisiológicas que en la tabla anterior se presentan, pueden servir como base para tratar de explicar o entender, los resultados que muestra el diagrama \#1. Si se analiza por separado cada uno de los movimientos realizados durante las pruebas, se puede deducir que tanto la relación entre sentadillasalto, $(2.1 \%)$ como la relación entre sentadilla 
- carreras de velocidad (5.1\%), las cuales son muy bajas pueden estar provocándose, debido a que ambos movimientos presentan, cargas desiguales para los diferentes grupos musculares que intervienen en la acción; donde en el salto y en la velocidad, el sujeto únicamente resiste su peso corporal; mientras que en la sentadilla completa la carga levantada es aproximadamente el doble del peso corporal, lo cual a su vez está provocando que la velocidad de movimiento en la sentadilla completa sea mucho más lenta, que la velocidad de ejecución que se puede alcanzar en las pruebas de salto. Otra diferencia entre estas pruebas es el ángulo de flexión que alcanza la articulación de la rodilla principalmente, en este caso la profundidad de la sentadilla completa provoca un ángulo de flexión mucho menor (de hasta $30^{\circ}$ ), mientras que en las pruebas de salto y velocidad, el ángulo de flexión es mayor $\quad\left(90^{\circ}-100^{\circ} \quad\right.$ aproximadamente) dependiendo de cada persona y tomando en cuenta que cada movimiento o repetición no es exactamente igual al anterior, aunque se den con un corto tiempo entre ambas. A partir de este análisis, se puede visualizar más fácilmente, algunas de las características en las que difieren la prueba de sentadilla con respecto a las pruebas de salto y velocidad, utilizadas en este estudio.

Tomando en cuenta estos razonamientos se puede concluir entonces, que podrían ser estas tres características, los pilares fisiológicos que la fibra muscular toma en cuenta a la hora de transferir su rendimiento en la ejecución de los diferentes tipos de pruebas.

Se debe tomar en cuenta que además de las características señaladas anteriormente, existen de hecho otros aspectos que no fueron contemplados en esta discusión y que pueden estar afectando el dificultoso análisis de los resultados obtenidos. Cabe señalar que en definitiva, las posibles explicaciones que se puedan buscar bajo estas condiciones de investigación, no son exhaustivas, por lo que no es conveniente asumir, que en el cuerpo humano, contemplado como un sistema sumamente complejo, el comportamiento de una fibra muscular aislada, puede ser explícitamente transferida al comportamiento de un grupo muscular en movimiento.

El hecho de que la práctica deportiva, esté basada en teoría que no está fundamentada en evidencia científicamente comprobable, hace que esta investigación resulte interesante, al cuestionar, hasta qué punto la teoría que se ha manejado por mucho tiempo está adaptada a las condiciones reales que se viven en la práctica. Por ejemplo, según los datos obtenidos en este estudio, no existe una razón por la que se debe de relacionar el concepto de fuerza máxima, expresado por medio de la prueba de $1 \mathrm{RM}$ de sentadilla completa con el de altura del salto vertical en la práctica, lo que difiere grandemente con Cometti (1998) quien afirma que la fuerza es la cualidad física fundamental, base de todas las demás capacidades condicionales, sin embargo, no se deben hacer aseveraciones del todo precisas, puesto que este autor no menciona en su texto, bajo qué condiciones hace estas afirmaciones, ni cuál fue el método que utilizó para llegar a esta conclusión. Baker (2001) afirma que a pesar de que el componente de potencia involucra tanto a la velocidad como a la fuerza, los entrenadores tradicionalmente concentran los programas de entrenamiento en el desarrollo de la fuerza, con la esperanza de que exista algún grado de transferencia entre estos componentes, estas aseveraciones difieren de los resultados encontrados en el presente estudio.

Un punto importante a considerar, es el hecho de pretender, a partir de la utilización de pruebas de campo, explicar una relación que parte de premisas puramente físicas. Es muy probable que las pruebas estén marcando reacciones fisiológicas directamente asociadas con la mecánica de movimiento de las pruebas, y no el constructo específico con el cuál se relaciona. Por esta razón, es de suma importancia que quede claro que no se están relacionando constructos puros (Fuerza- potencia, potencia-velocidad, etc) sino pruebas que se 
relacionan altamente con esos constructos. Bajo las condiciones antes mencionadas, es beneficioso, no hacer aseveraciones sobre relaciones puras entre fuerza - velocidad, fuerza - potencia y potencia - velocidad, ya que por las características de las pruebas de campo, éstas, no permiten observar la relación pura y estricta que se da entre ellas en condiciones de laboratorio, puesto que en las pruebas de campo influyen múltiples factores que hacen confusa las diferentes relaciones que supuestamente, la literatura respalda con respecto a los diferentes constructos.

Sin embargo en la práctica deportiva, esa es la forma de medir cada una de estas cualidades físicas, y no se puede obviar que si las pruebas tienen altas correlaciones estadísticas con estos constructos es porque bajo condiciones de campo, éstas, son opciones válidas y confiables, que pueden ser utilizadas durante los macro ciclos de entrenamiento, tomando en cuenta que al fin y al cabo lo más importante son los resultados que obtengan los atletas, en las competencias o diferentes situaciones reales de juego y no el comportamiento de una fibra muscular aislada.

A manera de conclusión, y tomando en cuenta todos los aspectos que se han discutido en este documento, es importante destacar:

1. Si bien es cierto existen estudios que reportan altas relaciones entre los constructos de fuerza-potencia $y$ velocidad, los resultados han sido recolectados bajo condiciones de laboratorio y con alta tecnología, medios que no poseemos en la práctica real, y que a su vez no se ajustan a las características reales de entrenamiento o competición, que viven los atletas diariamente, por lo que estudios como el presente representan una fuente de información más accesible a los entrenadores y personas que están directamente relacionadas con los deportistas.
2. Es importante no hablar en la práctica, de fuerza como tal, o potencia, sino de características mecánicas y fisiológicas del movimiento que permiten tener parámetros más objetivos de las diferentes cualidades físicas (velocidad de movimiento, carga, impacto, etc ) y cómo podemos mejorarlas.

3. Esta experiencia deja claro y latente que existe un abismo difícil de llenar entre la teoría y sus implicaciones en la práctica deportiva, por lo que no sólo es necesario que estudios como el presente busquen crear la ciencia del entrenamiento deportivo, sino que los teóricos e investigadores se preocupen por lograr ese acercamiento de la teoría a la práctica, planteando la literatura a partir de estudios que manipulen variables que estén más cercanas a la realidad que se vive en el ámbito deportivo.

4. Es urgente que en la literatura se incluyan metodologías y detalles acerca de los estudios en los que se sustentan, qué permitan corroborar los resultados propuestos, puesto que no es recomendable hacer aseveraciones, sin tomar en cuenta bajo que condiciones hacen dichas afirmaciones.

5. Es de gran interés, para quienes pretenden lograr mejorar el nivel deportivo en general, que se haga más investigación en esta línea, cuestionando y corroborando la información que se maneja en la actualidad. Así, una lectura cuidadosa de este documento, podría abrir opciones para obtener resultados más efectivos en campo deportivo.

\section{REFERENCIAS}

Aragón, L. F. (1994). Papel de la elasticidad del tendón en la potencia muscular. Memoria del I Congreso Internacional sobre entrenamiento deportivo. Editado por Universidad de Costa Rica.

Baker, D. y Nance, S. (1999). The relationship between running speed and measures of strength and power in professional rugby league players. 
Journal of Strength and Conditioning Research 13(3), 230-235.

Baker, D. (2001). The effects of an in-season of concurrent training on the maintenance of maximal strength and power in professional and college- aged rugby League Football players. Journal of Strength and Conditioning Research, 15(2), 172-177.

Bobbert, F., Huijing, P. \& Gerrit Jan Van, I. (1986). Drop jump.I. The influence of jumping technique on the biomechanics of jumping. Medicine and science sport and exercise. 19(4).

Bosco, C. (1985). Stretch-Shortening cycle in skeletal muscle function, and physiological consideration on explosive power in men. Atleticastudi, 1, 67-113.

Bosco, C. (1994). La valoración del test de Bosco. España. Editorial Paidotribo.

Cavagna,C.A., y Kaneco, M. (1976). Mechanical work and efficiency in level walking and running. Journal Physiology of London, 268, 467-481.

Cometti,G (1998) La Pliometría.. Barcelona España. INDE Publicaciones,

Díaz, J \& Molina, R (1994). Desarrollo de la fuerza, velocidad y potencia en los músculos extensores de la pierna: una comparación entre el entrenamiento pliométrico y pesas. Tesis para optar por el grado de licenciatura. Escuela de Educación Física y Deportes. Universidad de Costa Rica.

García-Manso, J. M., Navarro, M., Ruíz, -Caballero, J.A., Acero, R.M. (1998). La velocidad. Madrid.España. Editorial Gymnos.
Grosser, M., Starischka, S., y Zimmermann, E. (1988). Principios del entrenamiento deportivo. Barcelona. M. Roca.

Komi, P.V. (1992). Basics definitions of exercise. strength and power in sport. Germany. Blackwell scientific publications.

Miller B \& Power, S (1982). El salto en profundidad como medio de desarrollo de la potencia en atletismo. Cuaderno de Atletismo. Acondicionamiento físico deportivo. Real Federación Española de Atletismo.

Ortíz, C. V. (1996). Entrenamiento de fuerza y explosividad para la actividad física y el deporte de competición. Barcelona España.. INDE publicaciones.

Peralta, H. (1993). Hacia las posibilidades infinitas de la enseñanza de la gimnasia. Bogotá, Colombia. .Editorial C.I.P.

Pila Teleña, A. (1985) Preparación Física $3^{\circ}$ nivel. San José. Costa Rica. Editorial Olimpia.

Thomas, J \& Nelson, J (1990). Research Methods in Physical Activity. $\left(2^{\text {nd }}\right.$ ed) Illinois, USA. Human Kinetics.

Vega, J (1993). Efecto de dos tipos de entrenamiento pliométrico en el mejoramiento de la habilidad del salto vertical en jugadoras de fútbol universitarias. Memoria XIV Congreso Panamericano de Educación Física, vol. 1,79-86.

Verjoshanski, J.V. (1990). Entrenamiento deportivo, planificación y desarrollo. Barcelona España Editorial Martínez Roca, S.A.

Weineck, Jürgen. (1988). Entrenamiento óptimo. Barcelona España. Editorial Hispano Europea. 